\title{
Effect of Source, Surfactant, and Deposition Process on Electronic Properties of Nanotube Arrays
}

\author{
Dheeraj Jain, ${ }^{1}$ Nima Rouhi, ${ }^{1}$ Christopher Rutherglen, ${ }^{1}$ Crystal G. Densmore, ${ }^{2}$ \\ Stephen K. Doorn, ${ }^{2}$ and Peter J. Burke, \\ ${ }^{1}$ Integrated Nanosystems Research Facility, Department of Electrical Engineering and Computer Science, University of California, \\ Irvine, CA 92697, USA \\ ${ }^{2}$ Chemical Diagnostics and Engineering (C-CDE) Group, Chemistry Division, Los Alamos National Laboratory, Los Alamos, \\ NM 87545, USA \\ ${ }^{3}$ Department of Printed Electronics, Sunchon National University, Suncheon, Jeonnam 540-742, Republic of Korea
}

Correspondence should be addressed to Peter J. Burke, pburke@uci.edu

Received 6 May 2010; Accepted 27 September 2010

Academic Editor: Sulin Zhang

Copyright (C) 2011 Dheeraj Jain et al. This is an open access article distributed under the Creative Commons Attribution License, which permits unrestricted use, distribution, and reproduction in any medium, provided the original work is properly cited.

The electronic properties of arrays of carbon nanotubes from several different sources differing in the manufacturing process used with a variety of average properties such as length, diameter, and chirality are studied. We used several common surfactants to disperse each of these nanotubes and then deposited them on Si wafers from their aqueous solutions using dielectrophoresis. Transport measurements were performed to compare and determine the effect of different surfactants, deposition processes, and synthesis processes on nanotubes synthesized using CVD, CoMoCAT, laser ablation, and HiPCO.

\section{Introduction}

1.1. Motivation. Carbon nanotubes can be used for highperformance electronics [1] (defense), printed electronics [2] (commercial), and molecular sensors [3] (security) as well as a host of other potentially exotic applications such as nanoantennas $[4,5]$ and molecular beacons [6]. However, a scalable manufacturing technology for nanotube devices with appropriate yield, reproducibility, and performance in various metrics (mobility, on/off ratio, cost, chemical sensitivity, sensor specificity, etc.) is currently lacking. This is, in part, due to the variety of synthesis and deposition techniques and challenges due to the inherence in nanotube physical properties.

Of these challenges, nanotube dispersion in solution followed by deposition into arrays or mats that preserves the intrinsic, high performance is the largest, unmet challenge. Thus, there is a great need for an integrated, comprehensive study of the effect of nanotubes synthesis procedures, dispersion procedures, and deposition processes on the electronic properties of nanotube arrays. In this paper, we provide the first attempt at such a comprehensive study. While a complete understanding of the physical processes involved in these complex manufacturing steps is still lacking, our work provides the first, raw set of empirical guidelines to guide future technology development in this area.

1.2. Dispersion. It is well known that synthesized carbon nanotubes (especially single-walled carbon nanotubes SWNTs) exist in the form of bundles and that to exploit their electronic properties to the fullest; a postprocessing technology is required to separate them into individual nanotubes. A common approach to achieve this is by stabilizing the hydrophobic nature of nanotube surface with the use of a surfactant that overcomes the van der Waals forces among the nanotubes and results in suspensions of individual SWNTs.

It is also well known that all known synthesis methods for single-walled carbon nanotubes result in a mixture of chiralities and diameters, resulting in heterogeneous electrical properties, particularly a mixture of semiconducting and metallic nanotubes. In general, it is assumed to be an even distribution of folding chiralities, $1 / 3$ of all SWNTs in 
a sample being metallic with the remaining $2 / 3$ exhibiting semiconducting characteristics. The chemistry community has lead a successful effort aimed at sorting and purifying nanotubes in solution post-synthesis [7].

To date, a wide variety of surfactants have been reported to be able to disperse the nanotube in bundles into their individual form in an aqueous media successfully $[7,8]$. Several commercial surfactants such as sodium dodecyl sulfate (SDS), sodium cholate (SC), and sodium dodecylbenzenesulfonate (SDBS) are among the most reported for an efficient dispersion. Most dispersion studies have been directed toward chemical modification of the nanotube surface. Although many researchers have tried to solubilize nanotube ends and exterior walls through various functionalization routes [9], dispersion via functionalization has found limited success. Furthermore, covalent functionalization disturbs the extended $\pi$-electron systems ( $\mathrm{sp}^{2}$ orbitals) of the nanotube surface, responsible for many attractive attributes of SWNTs. Therefore, noncovalent surfactants are desired.

At present, it is not known how these surfactants are used since both the dispersion and metallic/semiconducting nanotube separation affect the electronic properties of nanotube arrays and films made from depositing nanotubes using various techniques. Adsorption of ionic surfactants on the surface could significantly modulate the device characteristics and affect the conductance of the devices [10]. It is critical to compare the electronic nature of the nanotube-surfactant conjugates versus intact nanotubes, since these reagents are increasingly being used in industry and laboratories. It is the purpose of this work to investigate the effect of various surfactant and deposition techniques on nanotube electronic properties using carbon nanotubes synthesized and purified using a variety of methods from a variety of academic and industrial institutions.

1.3. Dielectrophoresis. Several postsynthesis processes involve solution-based processing of nanotubes such as spin coating [11] or dielectrophoresis (DEP) deposition [12, 13]. Spincoating is probably the best long-term approach but is not yet mature enough for ultradense arrays. Dielectrophoresis allows selective alignment of nanotubes into controlled locations, and so it is used in this work as a test bed for systematic studies of the effect of alignment, source, and surfactants on electronic properties of nanotube arrays.

In DEP deposition steps, the presence of both metallic and semiconducting carbon nanotubes in solution is the main hurdle to form single-nanotube conductance channels. When an AC field is applied to the electrodes with nanotubes between the gap, the induced dipole moment of SWNTs interacts with nonuniform field resulting in a dielectrophoretic force which is influenced by the electronic properties of the SWNTs and in turn causes a separation of metallic and semiconducting nanotubes [14]. Assuming carbon nanotubes to be small cylindrical dipoles for simplifying the equations, the dielectrophoretic force can be expressed as $F \sim\left(\varepsilon_{p}-\varepsilon_{m}\right) / \varepsilon_{m}$, where $\varepsilon_{p}$ is the dielectric constant of the carbon nanotubes and $\varepsilon_{m}$ is the dielectric constant of the solvent [15]. At higher frequencies, this force is basically proportional to the difference between dielectric constant of the carbon nanotubes and dielectric constant of the solvent. The force observed by metallic nanotubes is significantly larger than that on semiconducting nanotubes because of the high dielectric constant of metallic SWNTs and the low dielectric constant of semiconducting SWNTs [14]. When a solvent with a dielectric constant between those values is used, separation of SWNTs will occur with metallic SWNTs attracted towards the field source (positive DEP) and semiconducting SWNTs repelled from the source (negative DEP). This physical effect causes separation of nanotubes due to metallic nanotubes being attracted towards field source electrodes while leaving behind semiconducting nanotubes in solution.

In this work, we use DEP for the deposition process. Therefore, our studies tend to elucidate arrays of metallic SWNTs primarily. However, these initial pioneering studies are meant to provide qualitative guidelines for the effects of surfactants and synthesis recipes on all types of SWNT arrays, including random and aligned arrays of all-semi, allmetal, or mixed species of SWNTs. Such studies are urgently needed if nanotube electronics is to progress from science to technology.

\section{Device Fabrication}

Single-walled carbon nanotubes were obtained from several different sources. Nanotubes obtained differ in terms of synthetic procedure used for their fabrication. Table 1 contains a list of the nanotube sources that we used with some details of their basic properties.

Nanotube diameters as mentioned in this table are obtained from the datasheets provided by their supplier and are also listed on their websites.

Each of these nanotubes (except semienriched samples from Los Alamos [16] and semienriched (90\% semiconducting) solution obtained from Nanointegris was first dispersed in surfactant solutions. Surfactants used for the comparison in our experiments were SDS, SC, and SDBS. The protocol used for the dispersion of each sample is as follows.

(1) $5 \% \mathrm{w} / \mathrm{v}$ solutions of surfactants were prepared in deionized water.

(2) $5 \mathrm{mg}$ of nanotube sample was dispersed in $10 \mathrm{~mL}$ of surfactant solution.

(3) Suspensions formed after addition of nanotubes were then sonicated for 3 hours.

(4) Mixtures were then centrifuged at $16,400 \mathrm{rpm}$ (at $30^{\circ} \mathrm{C}$ ) for 1 hour, 6 times repeatedly.

Dispersions obtained after 6 times centrifugation were then used for the deposition. Purified nanotubes obtained from Las Alamos were first dialyzed with 1\% SDS solution in deionized water prior to their use. Nanointegris nanotube solution was used as received.

Using a glass micropipette with a tip diameter of $\sim 20 \mu \mathrm{m}$, the supernatant was placed on the electrodes and DEP was used to accumulate the solubilized SWNTs within 
TABLe 1: Nanotube sources and their properties.

\begin{tabular}{lcc}
\hline Company & Synthetic process used & Avg. diameter as reported by provider \\
\hline CNI/Unidym & HiPCO & $0.81-0.85 \mathrm{~nm}$ \\
SouthWest Nanotechnologies & CoMoCAT & $0.8-1 \mathrm{~nm}$ \\
Carbon Solutions & Arc discharge & $1-1.4 \mathrm{~nm}$ \\
Las Alamos (semi-enriched) & HiPCO & $0.81-0.85 \mathrm{~nm}$ \\
Cheap Tubes & CVD & $1-2 \mathrm{~nm}$ \\
Nanointegris (semienriched, 90\%) & Arc discharge & $1.2-1.6 \mathrm{~nm}$ \\
\hline
\end{tabular}

TABLE 2: Summary of results.

\begin{tabular}{|c|c|c|c|c|}
\hline SWNT Sources & $\begin{array}{l}\text { Electrode } \\
\text { spacing }\end{array}$ & $\begin{array}{c}\text { Avg. length after } \\
\text { deposition (in SEM) }\end{array}$ & $\begin{array}{l}\text { DC resistance (1000's } \\
\text { of SWNTs in parallel) }\end{array}$ & On/off ratio \\
\hline Cheap Tubes & $3 \mu \mathrm{m}$ & $>3 \mu \mathrm{m}$ & $<50 \Omega$ & $2-8 \%$ \\
\hline SWeNT & $1 \mu \mathrm{m}$ & $<1 \mu \mathrm{m}$ & $50-100 \Omega$ & $2-6 \%$ \\
\hline CSI & $1 \mu \mathrm{m}$ & $<1.5 \mu \mathrm{m}$ & $100-200 \Omega$ & $<1 \%$ \\
\hline $\mathrm{CNI} /$ Unidym & $1 \mu \mathrm{m}$ & $<1 \mu \mathrm{m}$ & $100-500 \Omega$ & $<1 \%$ \\
\hline Las Alamos & $1 \mu \mathrm{m}$ & $<1.5 \mu \mathrm{m}$ & $120-150 \Omega$ & $<1 \%$ \\
\hline Nanointegris (semi-enriched, 90\%) & $1 \mu \mathrm{m}$ & $<1.5 \mu \mathrm{m}$ & $150-180 \Omega$ & $<1 \%$ \\
\hline
\end{tabular}

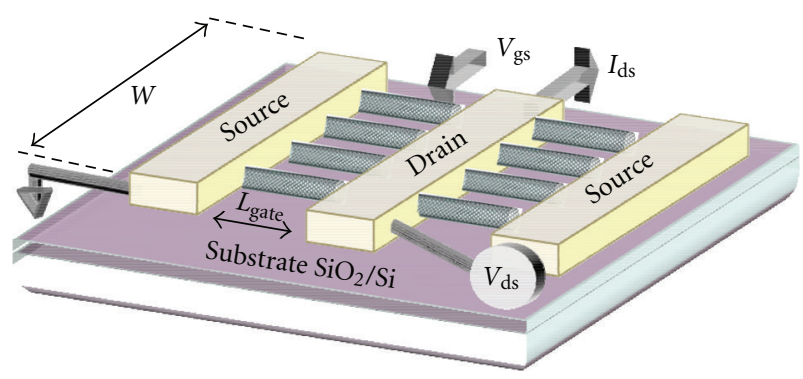

Figure 1: Diagram of RF probing and dual coplanar waveguide/electrode structure.

the electrode gap. This was accomplished using a $1-5 \mathrm{~V}_{\mathrm{pp}}$, $300 \mathrm{KHz}-25 \mathrm{MHz}$ sine wave voltage signal applied to the electrodes. The drop was then air dried, and the residue from the solution left after evaporation was gently washed away with deionized water.

Figure 1 shows the layout for our measurements. Device fabrication for these experiments was done using a similar protocol described in our earlier work [13]. Using electron beam lithography, electrodes with a pair of gaps were patterned and evaporated with Ti/Au $5 \mathrm{~nm} / 50 \mathrm{~nm}$ on high resistivity Si wafers $(8000 \Omega-\mathrm{cm})$ with a $500 \mathrm{~nm}$ thermal oxide layer. Coplanar waveguide electrode geometries were patterned with photolithography and evaporated with $25 \mathrm{~nm}$ $\mathrm{Ti} / 250 \mathrm{~nm}$ Au to form a dual waveguide/electrode structure with a pair of electrode gaps and with widths of $100 \mu \mathrm{m}$. After accumulation of aligned SWNTs within the gap by DEP, as described further in this paper, contact electrodes were patterned using e-beam lithography and evaporated with $70 \mathrm{~nm}$ of Pd producing a new electrode gap of $1 \mu \mathrm{m}$. The devices were RF-contacted electrically using a commercially available probe. Figure 2 shows a schematic diagram of

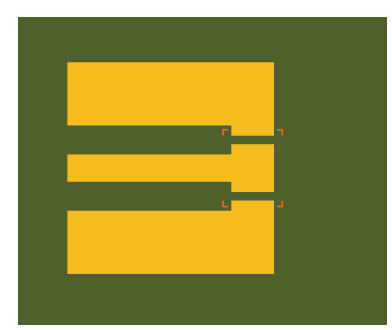

1) Coplanar waveguide on wafers

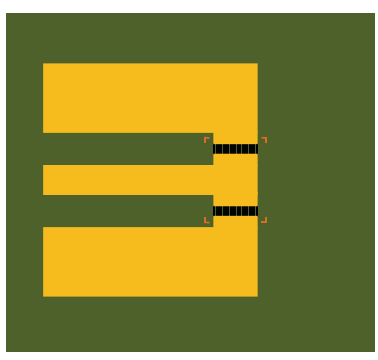

3) Dielectrophoresis: accumulate CNTs within electrode gap using dielectrophoresis

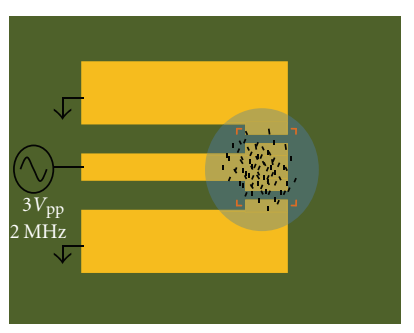

2) Placing solubilized CNTs on to electrodes

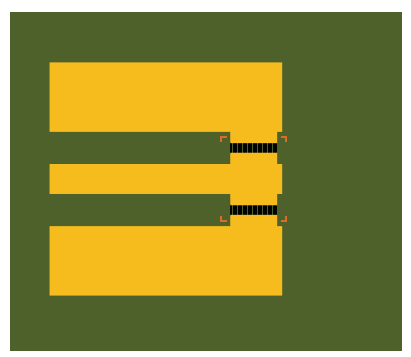

4) Extension electrodes: reduce gap width by e-beam patterning and evaporating extension electrodes
FIgURE 2: Schematic diagram of steps used in deposition of nanotubes on Si wafers.

several steps involved in the process of making a device with nanotubes deposited in the gaps.

Rather than characterize each device in full detail, we opted for a simplified, higher throughput measurement procedure in order to maximize the number of deposition conditions we could perform. For all the DC resistance measurements, a low source-drain bias voltage $(+1 \mathrm{~V}$ to $-1 \mathrm{~V}$ ) was used. Gate voltages of $-9,0$ and $+9 \mathrm{~V}$ were applied to the Si Substrate (bottom gate) to modulate the 


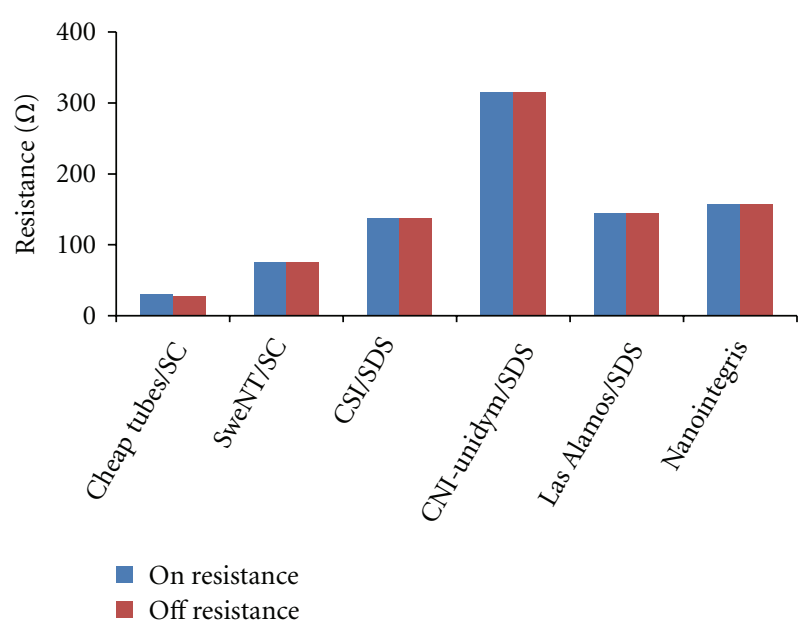

FIGURE 3: Results of DC resistance measurements done on nanotubes obtained from various sources that differ in synthetic process used (HiPCO/CoMoCat/Arc/CVD).

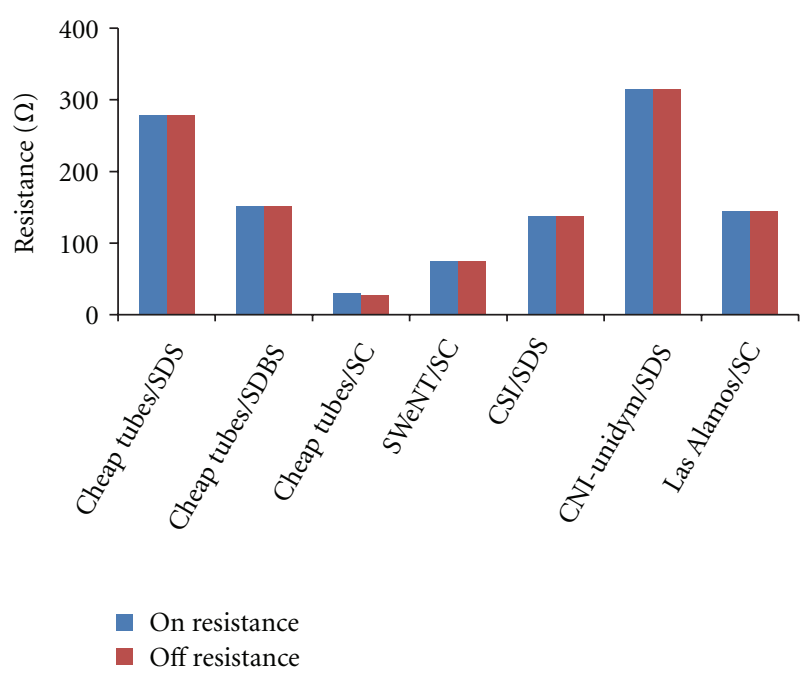

FIgURE 4: Results of DC resistance measurements done on nanotubes solubilized using variety of surfactants SDS, SC, and SDBS.

conductance and measure the on/off ratio for the nanotube devices. Because the full depletion curve was not measured, the on/off ratio is qualitative, not quantitative. (The devices may not have been completely "on" or "off" in our simplified measurement scheme.)

\section{Experimental Results}

Electronic measurements were performed on several devices fabricated by keeping some factors common for comparison. Based on our experiments, we have identified the role of frequency and amplitude used in DEP for the deposition of nanotubes. We were able to optimize these factors to make such device fabrication reproducible to a certain extent. Studies shown in this article would help researchers to establish a similar protocol for optimized deposition when nanotubes are obtained from different sources.

The following comparison results are based on the data obtained by averaging results for several similarly formed devices. DC-resistance values are normalized for a device with a width of $100 \mu \mathrm{m}$. The length of devices was kept in the range of the length of nanotubes from respective sources (Table 2). This would ensure that the comparison is based on the nanotubes similarly distributed over the length of devices and not over the width.

3.1. Comparison on Nanotube Sources. Nanotubes obtained from various sources were deposited on wafers, and electronic measurements done for them are summarized below. Nanotubes obtained from Unidyn (formerly Carbon Nanotechnologies Inc, HiPCO, 0.81-0.85 nm diameter, length $<1 \mu \mathrm{m}$ ) were found to show greater resistance as compared to the lowest shown by nanotubes from Cheap Tubes Inc. (Arc discharge, $1-2 \mathrm{~nm}$ diameter, length $>3 \mu \mathrm{m}$ ). Figure 3 shows a cumulative chart on DC resistance studies done on nanotubes that differ in their synthetic process. For our experiments, we obtained nanotubes from different sources to accommodate nanotubes produced by HiPCO, CoMoCAT, Arc, and CVD methods. In these experiments, $4 \mathrm{~V}_{\mathrm{pp}}, 25 \mathrm{MHz}$ sine wave voltage signal was applied for 3 minutes to the electrodes for the deposition of nanotubes.

3.2. Comparison on Using Different Surfactants. Three different surfactants-sodium cholate (SC), sodium dodecyl sulfate (SDS), and sodium dodecyl benzene sulfonate (SDBS) were used to solubilize nanotubes in aqueous solution. SC was found to be the most effective surfactant for solubilizing most of the nanotubes. Figure 4 shows the on/off DC resistance of nanotube devices where nanotubes from several sources were dissolved using different surfactants and deposited on wafers using DEP. In all the experiments, $4 \mathrm{~V}_{\mathrm{pp}}$, $25 \mathrm{MHz}$ sine wave voltage signal was applied for 3 minutes to the electrodes for the deposition of nanotubes.

3.3. Comparison on Using Different Frequencies. When different frequencies were used for the deposition, it was observed that nanotubes deposited using a frequency less than $15 \mathrm{MHz}$ were not aligned, while when a frequency above $25 \mathrm{MHz}$ was used, deposited nanotubes were found to be well aligned. Figure 5 shows some representative images of nanotubes deposited under various different conditions. Several different patterns of alignments owing to different DEP parameters were observed. In one case, it was also observed that when a nanotube solution (nanotube dispersed in $1 \% \mathrm{w} / \mathrm{v}$ solution of SC) was left standing prior to its use for DEP deposition for several days, nanotubes formed bigger bundles, which were apparent in their SEM images.

3.4. Comparison on Using Different Amplitude. Nanotube samples (Cheap Tubes, dissolved using SC) were deposited by applying several different voltages for DEP at a constant frequency of $25 \mathrm{MHz}$. In general, it was observed that when an amplitude above $3 \mathrm{~V}$ was applied, 2 minutes or more 


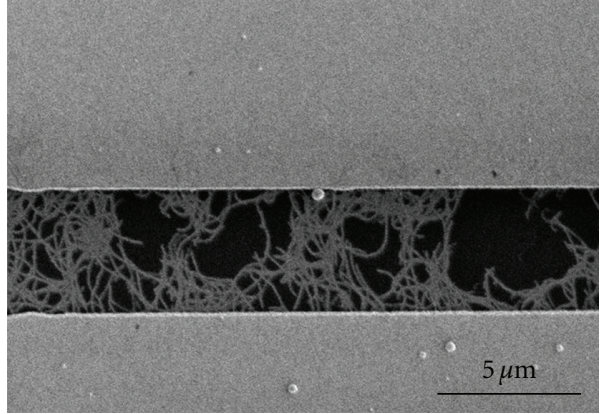

(a)

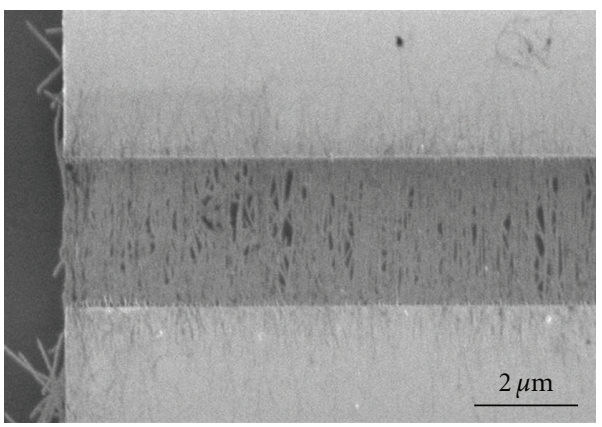

(c)

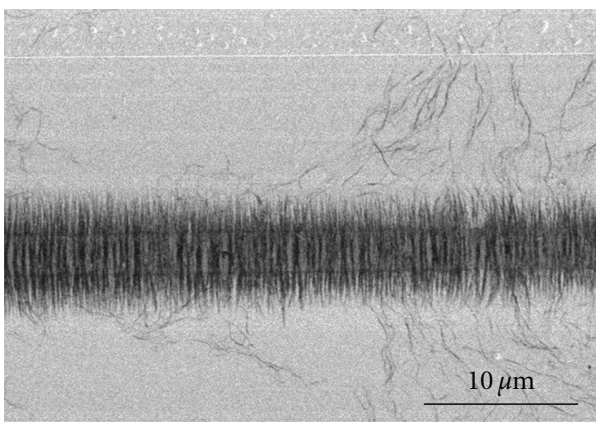

(e)

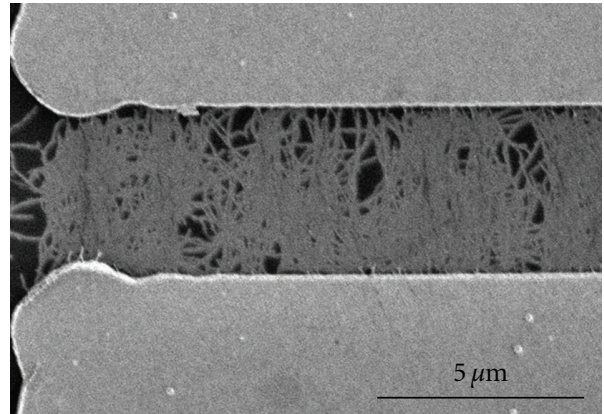

(b)

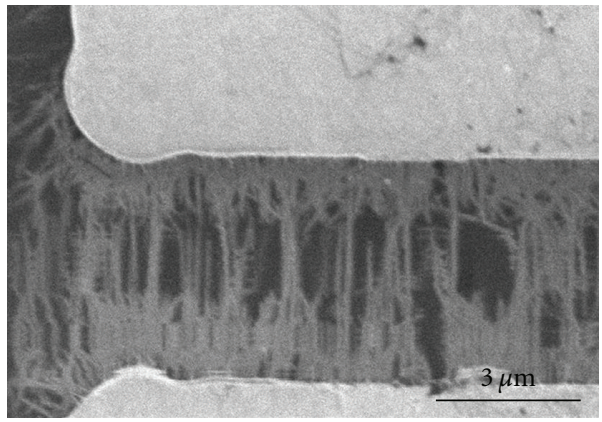

(d)

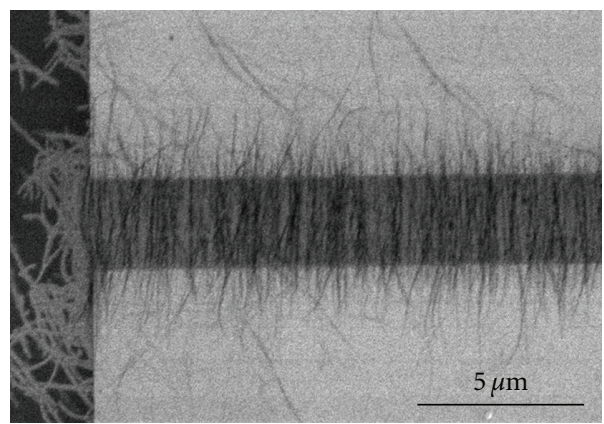

(f)

Figure 5: Nanotubes (Cheap Tubes) deposited on Si wafers at $2 \mathrm{MHz}$ (Figures 5(a), 5(b)) and $25 \mathrm{MHz}$ (Figures 5(c), 5(d)). The difference in their alignment is apparent. Figure 5(d) represents deposited nanotubes (at $25 \mathrm{MHz}$ ) shorter in length $(<2 \mu \mathrm{m})$; it can be seen that even shorter nanotubes were amazingly aligned in the gap. Figures 5(e), 5(f) show nanotubes deposited from a solution that was sonicated for a short time $(5 \mathrm{~min})$ and then left standing for a few days prior to use; formation of bigger bundles of nanotubes is apparent in the images.

was sufficient to fill the gap up to almost $100 \%$. When a voltage less than 1 was used, no nanotubes were apparently deposited. Using a dilute solution and giving more time for the deposition or using a comparatively concentrated solution with less time yielded the devices with similar properties. Also using any amplitude above $3 \mathrm{~V}$ did not make much difference on device characteristics.

Figure 7 shows a typical Raman spectrum from a device (with nanotubes from Cheap Tubes), indicating that indeed SWNTs are being deposited.
3.5. Reproducibility. In Figure 8 is shown a histogram plotted for DC-resistance measurement done on several devices that had different gap width. Most of the devices having the same width had shown DC resistance in similar range (Figure $8(\mathrm{a})$ ), which shows the reproducibility of our experiments. Figure 8(b) is a plot of averaged DC resistance observed for the devices plotted against their width. Increasing the width 10 times in fact lowered the resistance with an order of 10 too, which shows the reliability of the process in terms of reproducing the results over the repeated experiments. 


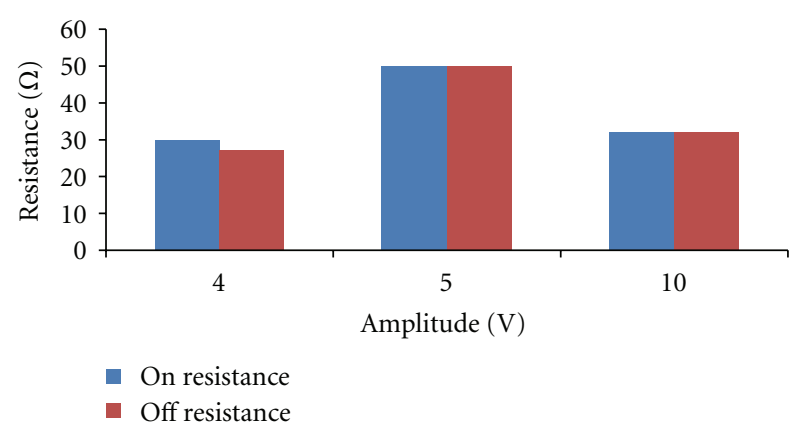

FIGURE 6: DC-resistance measurements done on nanotubes devices fabricated by depositing nanotubes using different amplitudes.

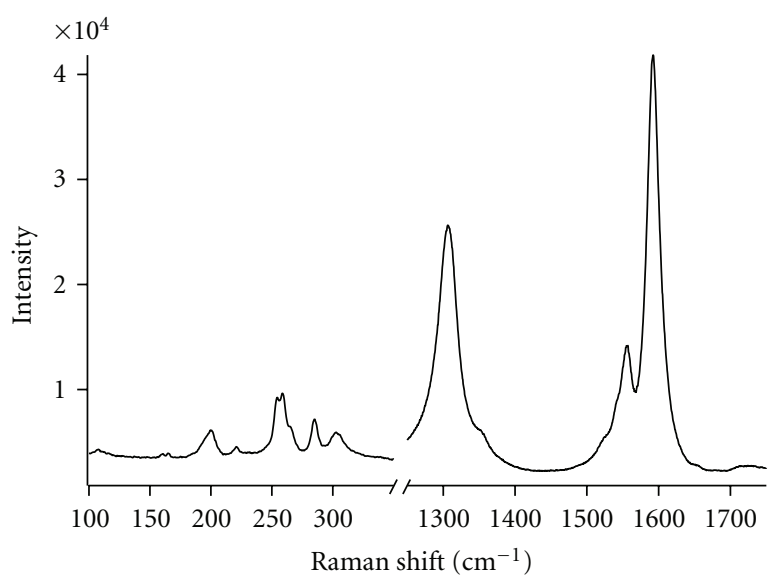

Figure 7: A typical Raman spectra from a device representative to the one shown in Figure 6 confirm the presence of nanotubes.

\section{Discussion}

The use of ionic surfactant to solubilized nanotubes is known to modulate the surface conductance of nanotubes that will eventually affect the dielectrophoresis force applied for the deposition of nanotubes. Strano's group studied effects of surface conductivity of semiconducting SWNTs induced by ionic surfactants on the sign of dielectrophoretic force [17]. They were able to modulate the surface conductance by changing the ionic strength of medium. Also, by neutralizing the surface charge using an equimolar mixture of anionic and cationic surfactant, they observed negative DEP of semiconducting species at $10 \mathrm{MHz}$. They suggested $10 \mathrm{MHz}$ to be the crossover frequency for their semiconducting nanotube. From our experiments we found that in most of the cases when nanotubes were deposited at frequencies higher than $5 \mathrm{MHz}$, only metallic behavior of deposited nanotubes was observed in their electronic measurements. Even when the solution enriched in semiconducting nanotube was used, dielectrophoretic deposition at any frequencies higher than $5 \mathrm{MHz}$ resulted in devices with extremely low or no on/off ratio. We believe this is because even if above crossover frequencies, semiconducting nanotubes might have observed positive DEP, amount of metallic nanotubes that were deposited in the process was much larger than semiconducting nanotubes.

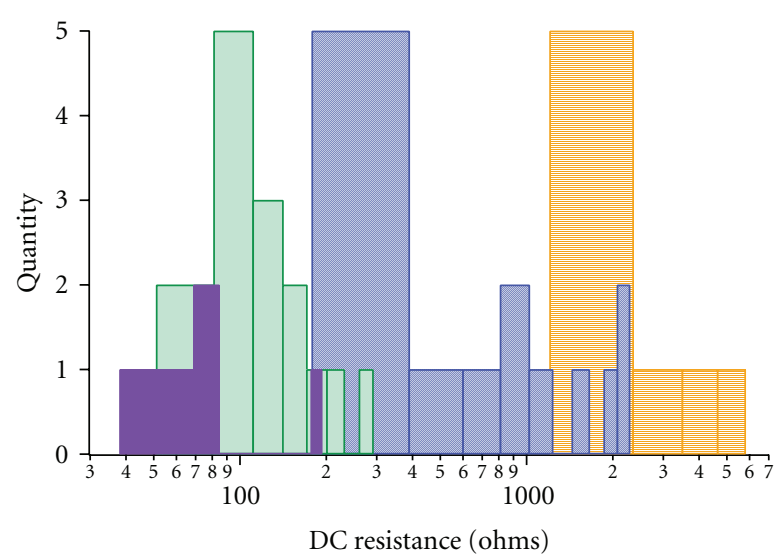

$$
\begin{aligned}
& \text { ㅌ } 5 \mu \mathrm{m} \\
& \text { 에 } 20 \mu \mathrm{m} \\
& \text { 뜾 } 100 \mu \mathrm{m} \\
& \text { 四 } 300 \mu \mathrm{m}
\end{aligned}
$$

(a)

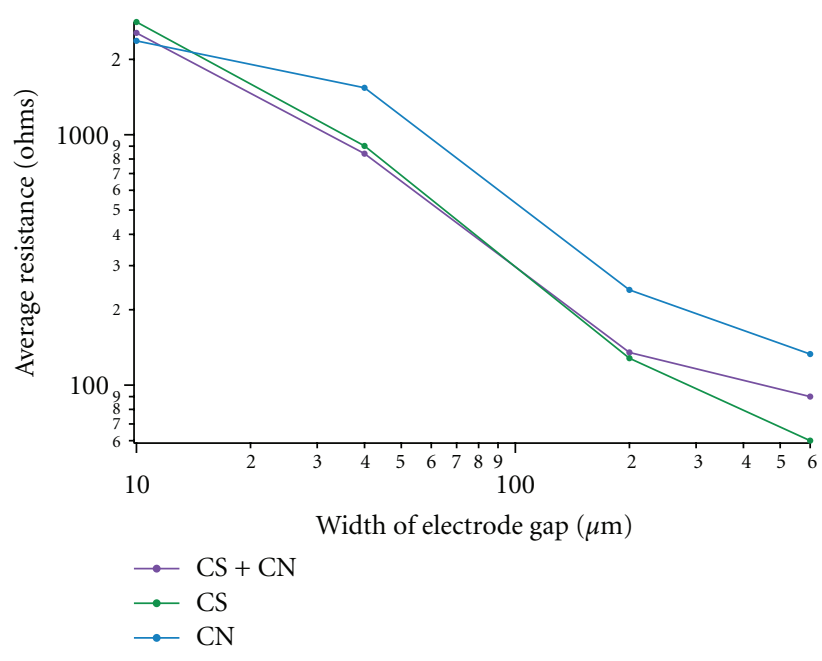

(b)

FIGURE 8: An averaged DC-resistance measurement done on devices having different electrode width.

The on/off ratios reported here are consistent with Happy et al. who found that when a conventional SWNT solution with $\sim 2 / 3$ of semiconducting nanotubes is deposited using DEP, major part of the DC current (90-95\%) is produced by metallic nanotubes $[18,19]$. They also found that when $99 \%$ semienriched solution was used for deposition with DEP along with substrate's surface modification, the devices did not go to complete off state, most likely because a small number of metallic nanotubes were preferentially deposited by DEP [19]. In another recent experiments [20], we used same semienriched solution (90\%, "IsoNanotubesS", Nanointegris) and deposited them without DEP by using only surface modification [21] which resulted in devices with better mobility and also higher on/off ratios than the current DEP method. An on/off ratio of up to 110000 was achieved when nanotubes were deposited using only surface modification. 


\section{Conclusion}

We have performed the first systematic study of the effects of surfactants, source, and deposition procedure on the electronic properties of nanotube devices. From our experience we believe, neither the surfactant used nor the source has a significant impact on the on- resistance. The alignment is much affected by the frequency of the AC voltage used to align the nanotubes. On the other hand, a consistent scaling of device resistance with width is observed for samples from different sources, indicating that the procedure can be empirically adjusted to give reasonably tight tolerances. Using this technique, the on/off ratio was never large, indicating that in most of the cases, metallic nanotubes were deposited preferentially. Finally, we consistently (with almost perfect yield) were able to achieve devices with resistance of order $50 \Omega$, regardless of nanotubes source or surfactant, which holds promise for future RF devices with nanotube arrays.

\section{Acknowledgments}

The authors would like to thank Jie Liu from Duke University for Raman spectroscopy. This work was funded by the National Science Foundation, Army Research Office, Office of Naval Research, Northrop Grumman, and Korean National Science Foundation (KOSEF) World Class University (WCU) program.

\section{References}

[1] C. Rutherglen, D. Jain, and P. Burke, "Nanotube electronics for radiofrequency applications," Nature Nanotechnology, vol. 4, pp. 811-819, 2009.

[2] Q. Cao and J. A. Rogers, "Ultrathin films of single-walled carbon nanotubes for electronics and sensors: a review of fundamental and applied aspects," Advanced Materials, vol. 21, no. 1, pp. 29-53, 2009.

[3] P. G. Collins, K. Bradley, M. Ishigami, and A. Zettl, "Extreme oxygen sensitivity of electronic properties of carbon nanotubes," Science, vol. 287, no. 5459, pp. 1801-1804, 2000.

[4] P. J. Burke, S. Li, and Z. Yu, "Quantitative theory of nanowire and nanotube antenna performance," IEEE Transactions on Nanotechnology, vol. 5, no. 4, pp. 314-334, 2006.

[5] P. J. Burke, C. Rutherglen, and Z. Yu, "Carbon nanotube antennas," in Proceedings of the 9th International Conference on Electromagnetics in Advanced Applications, p. 937, 2005.

[6] H. Jin, D. A. Heller, and M. S. Strano, "Single-particle tracking of endocytosis and exocytosis of single-walled carbon nanotubes in NIH-3T3 cells," Nano Letters, vol. 8, no. 6, pp. 1577-1585, 2008.

[7] M. C. Hersam, "Progress towards monodisperse single-walled carbon nanotubes," Nature Nanotechnology, vol. 3, no. 7, pp. 387-394, 2008.

[8] V. C. Moore, M. S. Strano, E. H. Haroz et al., "Individually suspended single-walled carbon nanotubes in various surfactants," Nano Letters, vol. 3, no. 10, pp. 1379-1382, 2003.

[9] C. A. Dyke and J. M. Tour, "Covalent functionalization of single-walled carbon nanotubes for materials applications," Journal of Physical Chemistry A, vol. 108, no. 51, pp. 1115111159, 2004.
[10] Q. Fu and J. Liu, "Effects of ionic surfactant adsorption on single-walled carbon nanotube thin film devices in aqueous solutions," Langmuir, vol. 21, no. 4, pp. 1162-1165, 2005.

[11] M. C. LeMieux, M. Roberts, S. Barman, W. J. Yong, M. K. Jong, and Z. Bao, "Self-sorted, aligned nanotube networks for thinfilm transistors," Science, vol. 321, no. 5885, pp. 101-104, 2008.

[12] R. Krupke, F. Hennrich, H. B. Weber, M. M. Kappes, and H. V. Löhneysen, "Simultaneous deposition of metallic bundles of single-walled carbon nanotubes using ac-dielectrophoresis," Nano Letters, vol. 3, no. 8, pp. 1019-1023, 2003.

[13] C. Rutherglen, D. Jain, and P. Burke, "Rf resistance and inductance of massively parallel single walled carbon nanotubes: direct, broadband measurements and near perfect 50 impedance matching," Applied Physics Letters, vol. 93, no. 8, Article ID 083119, 2008.

[14] R. Krupke, F. Hennrich, H. V. Löhneysen, and M. M. Kappes, "Separation of metallic from semiconducting single-walled carbon nanotubes," Science, vol. 301, no. 5631, pp. 344-347, 2003.

[15] M. Dimaki and P. Bøggild, "Dielectrophoresis of carbon nanotubes using microelectrodes: a numerical study," Nanotechnology, vol. 15, no. 8, pp. 1095-1102, 2004.

[16] S. Niyogi, C. G. Densmore, and S. K. Doorn, "Electrolyte tuning of surfactant interfacial behavior for enhanced densitybased separations of single-walled carbon nanotubes," Journal of the American Chemical Society, vol. 131, no. 3, pp. 1144$1153,2009$.

[17] Y. Kim, S. Hong, S. Jung, M. S. Strano, J. Choi, and S. Baik, "Dielectrophoresis of surface conductance modulated single-walled carbon nanotubes using catanionic surfactants," Journal of Physical Chemistry B, vol. 110, no. 4, pp. 1541-1545, 2006.

[18] A. Le Louarn, F. Kapche, J.-M. Bethoux et al., "Intrinsic current gain cutoff frequency of $30 \mathrm{GHz}$ with carbon nanotube transistors," Applied Physics Letters, vol. 90, no. 23, Article ID 233108, 2007.

[19] L. Nougaret, H. Happy, G. Dambrine et al., " $80 \mathrm{GHz}$ fieldeffect transistors produced using high purity semiconducting single-walled carbon nanotubes," Applied Physics Letters, vol. 94, no. 24, Article ID 243505, 2009.

[20] N. Rouhi, D. Jain, K. Zand, and P. J. Burke, "Allsemiconducting nanotube devices for RF and microwave applications," in Proceedings of the IEEE MTT International Microwave Symposium, May 2009.

[21] S. Auvray, V. Derycke, M. Goffman, A. Filoramo, O. Jost, and J.-P. Bourgoin, "Chemical optimization of self-assembled carbon nanotube transistors," Nano Letters, vol. 5, no. 3, pp. 451-455, 2005. 

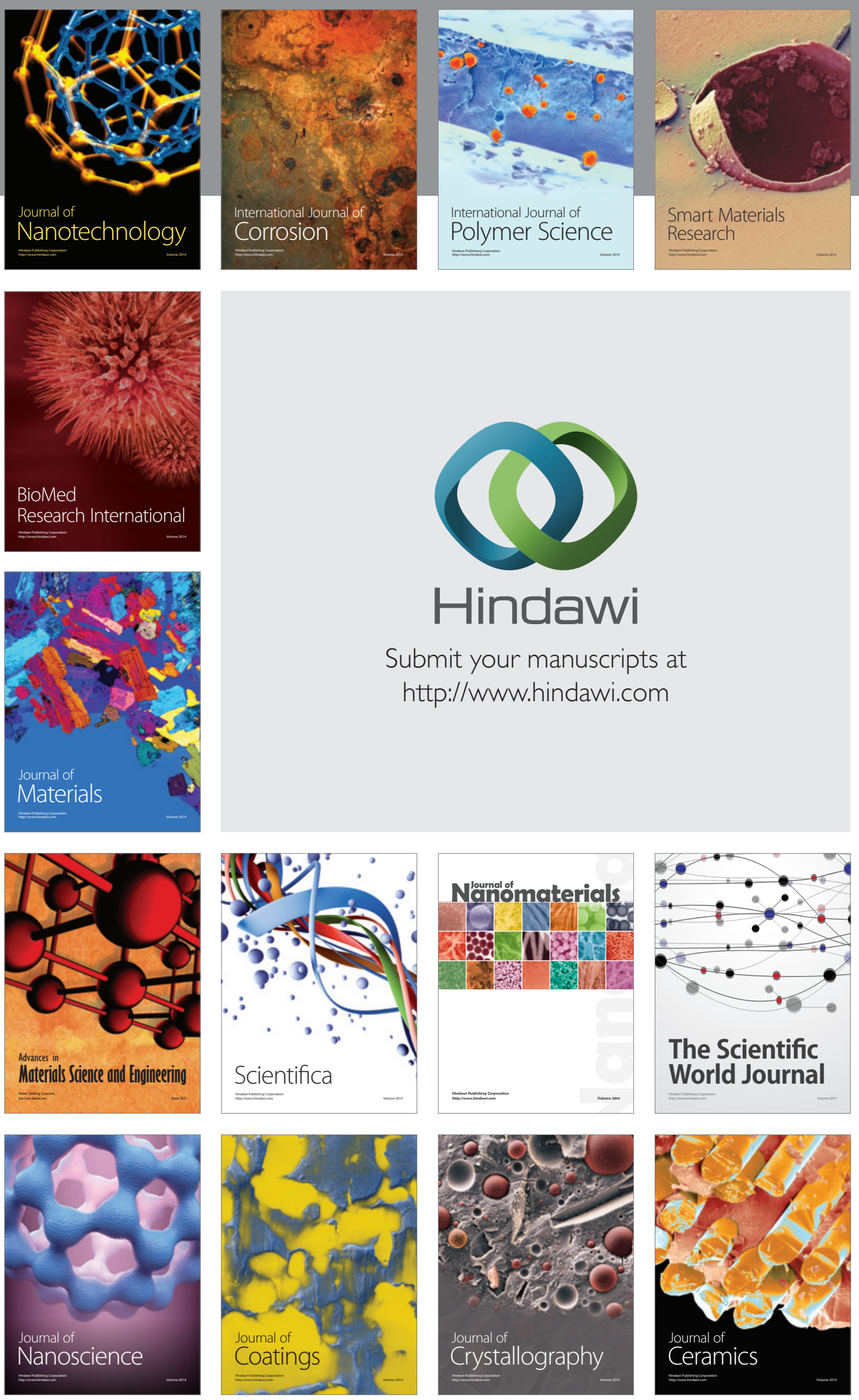

The Scientific World Journal

Submit your manuscripts at

http://www.hindawi.com

\section{World Journal}

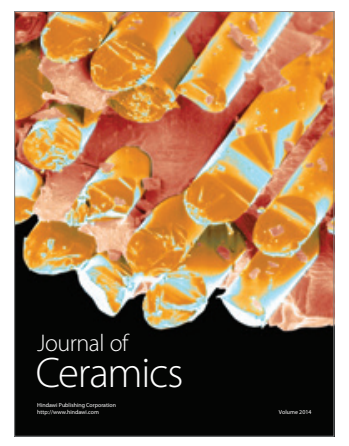

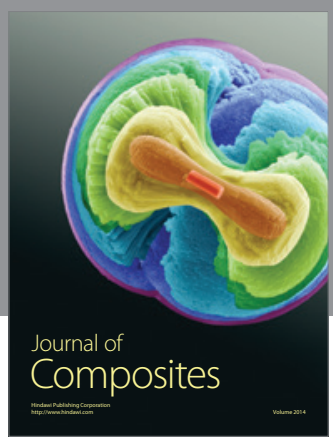
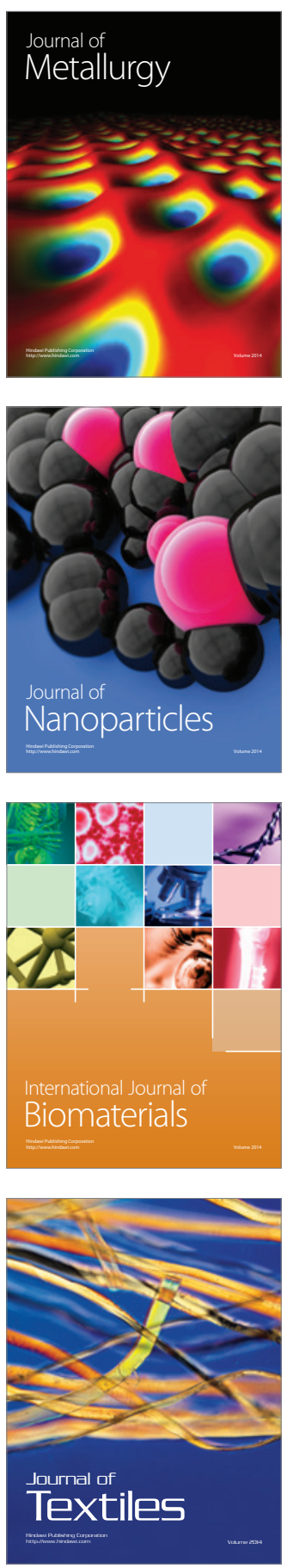